\title{
The $q$-Binomial Theorem and two Symmetric $q$-Identities
}

\author{
Victor J. W. Guo \\ Center for Combinatorics, LPMC \\ Nankai University, Tianjin 300071, People's Republic of China \\ jwguo@eyou.com
}

Submitted: Jun 30, 2003; Accepted: Sep 4, 2003; Published: Sep 12, 2003

MR Subject Classifications: 05A19, 05A17

\begin{abstract}
We notice two symmetric $q$-identities, which are special cases of the transformations of ${ }_{2} \phi_{1}$ series in Gasper and Rahman's book (Basic Hypergeometric Series, Cambridge University Press, 1990, p. 241). In this paper, we give combinatorial proofs of these two identities and the $q$-binomial theorem by using conjugation of 2-modular diagrams.
\end{abstract}

\section{Introduction}

We follow the notation and terminology in [7], and we always assume that $0 \leq|q|<1$. The $q$-shifted factorial is defined by

$$
(a ; q)_{0}=1, \quad(a ; q)_{n}=\prod_{k=0}^{n-1}\left(1-a q^{k}\right), n \in \mathbb{N}, \quad(a ; q)_{\infty}=\prod_{k=0}^{\infty}\left(1-a q^{k}\right) .
$$

The following theorem is usually called the q-binomial theorem. It was found by Rothe, and was rediscovered by Cauchy (see $[1$, p. 5]).

Theorem 1.1 If $|z|<1$, then

$$
\sum_{n=0}^{\infty} \frac{(a ; q)_{n}}{(q ; q)_{n}} z^{n}=\frac{(a z ; q)_{\infty}}{(z ; q)_{\infty}}
$$

Various proofs (1.1) are known. For simple proofs of (1.1), see Andrews [3, Section 2.2] and Gasper [6], and for combinatorial proofs, see Alladi [2] and Pak [8].

The following two theorems are special cases of the transformations of ${ }_{2} \phi_{1}$ series in Gasper and Rahman [7, p. 241]. 
Theorem 1.2 For $|a|<1$ and $|b|<1$, we have

$$
\sum_{n=0}^{\infty} \frac{(a z ; q)_{n}}{(a ; q)_{n+1}} b^{n}=\sum_{n=0}^{\infty} \frac{(b z ; q)_{n}}{(b ; q)_{n+1}} a^{n}
$$

Theorem 1.3 We have

$$
\sum_{k=0}^{n} \frac{(q / z ; q)_{k}(z ; q)_{n-k}}{(q ; q)_{k}(q ; q)_{n-k}} q^{m k} z^{k}=\sum_{k=0}^{m} \frac{(q / z ; q)_{k}(z ; q)_{m-k}}{(q ; q)_{k}(q ; q)_{m-k}} q^{n k} z^{k}
$$

Clearly, the left-hand side of (1.2) may be written as

$$
\frac{1}{(1-a)}{ }_{2} \phi_{1}(a z, q ; q a ; q, b) \text {. }
$$

By the Heine's transformation (III.1) in Gasper and Rahman [7, p. 241], (1.4) is equal to

$$
\frac{1}{(1-a)} \frac{(q, a b z ; q)_{\infty}}{(q a, b ; q)_{\infty}} \phi_{1}(a, b ; a b z ; q, q)
$$

which is symmetric in $a$ and $b$. Note that the special case $z=0$ of (1.2) has also appeared in the literature (see Stockhofe [9] and Pak [8, 2.2.4]).

Rewrite the left-hand side of (1.3) as

$$
\frac{(z ; q)_{n}}{(q ; q)_{n}}{ }_{2} \phi_{1}\left(q^{-n}, q / z ; q^{1-n} / z ; q, q^{m+1}\right)
$$

Applying the transformation (III.6) in [7, p. 241], we get

$$
q_{3}^{m n} \phi_{2}\left(q^{-n}, q^{-m}, z ; q, 0 ; q, q\right)
$$

which is symmetric in $m$ and $n$.

The purpose of this paper is to give combinatorial proofs of $(1.1),(1.2)$, and (1.3) by using conjugation of 2-modular diagrams.

As usual, a partition $\lambda$ is defined as a finite sequence of nonnegative integers $\left(\lambda_{1}, \lambda_{2}, \ldots\right.$, $\left.\lambda_{m}\right)$ in decreasing order $\lambda_{1} \geq \lambda_{2} \geq \cdots \geq \lambda_{m}$. A nonzero $\lambda_{i}$ is called a part of $\lambda$. The numbers of odd parts and even parts of $\lambda$ are denoted by odd $(\lambda)$ and even $(\lambda)$, respectively. Define $\ell(\lambda)=\operatorname{odd}(\lambda)+\operatorname{even}(\lambda)$, called the length of $\lambda$. Write $|\lambda|=\sum_{i=1}^{m} \lambda_{i}$, called the weight of $\lambda$.

The set of all partitions into even parts is denoted by $\mathcal{P}_{\text {even }}$. The set of all partitions into distinct odd parts is denoted by $\mathcal{D}_{\text {odd }}$. Let $\mathcal{P}_{1}$ (respectively, $\mathcal{P}_{2}$ ) denote the set of partitions with no repeated odd (respectively, even) parts.

For partitions $\lambda$ and $\mu$, we define $\lambda \cup \mu$ to be the partition obtained by putting all parts of $\lambda$ and $\mu$ together in decreasing order. 


\section{A Theorem on Partitions}

The following theorem is crucial to prove Theorems 1.1-1.3 combinatorially.

Theorem 2.1 Given $m \geq 1$, the number of partitions of $n$ into at most $m$ parts with no repeated odd parts is equal to the number of partitions of $n$ with the largest part at most $2 m$ and with no repeated odd parts.

Theorem 2.1 was established by Chapman [5] in his proof of the $q$-identity

$$
\sum_{n=1}^{\infty} n \frac{-q^{2 n-1}+q^{2 n}}{1-q^{2 n}} \prod_{j=1}^{n-1} \frac{1-q^{2 j-1}}{1-q^{2 j}}=\prod_{j=1}^{\infty} \frac{1-q^{2 j-1}}{1-q^{2 j}} \sum_{d=1}^{\infty}(-1)^{d} \frac{q^{d}}{1-q^{d}},
$$

which is due to Andrews, Jiménez-Urroz, and Ono [4]. Here we describe Chapman's proof.

Proof of Theorem 2.1. We shall construct an involution $\sigma$ on $\mathcal{P}_{1}$ such that $\sigma$ preserves $|\lambda|$ while interchanging $\ell(\lambda)$ and $\left\lceil\lambda_{1} / 2\right\rceil$.

We construct a diagram for each $\lambda \in \mathcal{P}_{1}$. Each part $\lambda_{i}$ will yield a row of length $\left\lceil\lambda_{i} / 2\right\rceil$. An even part $2 k$ will give a row of $k 2$ 's, while an odd part $2 k+1$ will give a row of $k 2$ 's followed by a 1 . Such a diagram is called a 2-modular diagram. As an example, let $\lambda=(10,9,7,4,4,4,3,2,2,1)$. Then, $\lambda$ gives the 2 -modular diagram

$\begin{array}{lllll}2 & 2 & 2 & 2 & 2 \\ 2 & 2 & 2 & 2 & 1 \\ 2 & 2 & 2 & 1 & \\ 2 & 2 & & & \\ 2 & 2 & & & \\ 2 & 2 & & \\ 2 & 1 & & \\ 2 & & & \\ 2 & & & & \\ 1 & & & & \end{array}$

Since no odd part of $\lambda$ is repeated, the 1's can only occur at the bottom of columns. We identify elements of $\mathcal{P}_{1}$ with their diagrams, and then define $\sigma$ to be conjugation of diagrams. For the above $\lambda, \sigma(\lambda)$ gives the 2-modular diagram

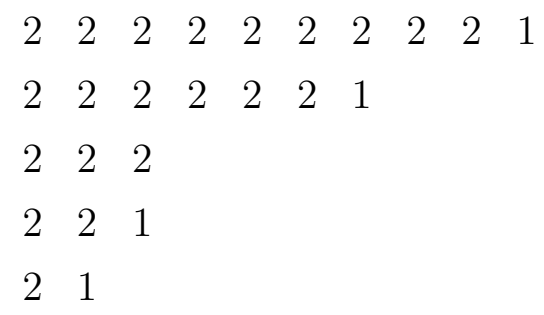

THE ELECTRONiC JOURNAL OF COMBinAtorics 10 (2003), \#R34 
Namely, $\sigma(\lambda)=(19,13,6,5,3)$. Clearly, the number of rows in the diagram of $\lambda$ is $\ell(\lambda)$, while the number of columns is $\left\lceil\lambda_{1} / 2\right\rceil$. Thus, $\sigma$ has the required properties and Theorem 2.1 is proved.

Note that the above involution $\sigma$ on $\mathcal{P}_{1}$ also preserves odd $(\lambda)$.

\section{Combinatorial Proofs of Theorems 1.1, 1.2, and 1.3}

In this section, we give combinatorial proofs of the $q$-binomial theorem and Theorems 1.2 and 1.3. Our combinatorial proof of the $q$-binomial theorem is based on Theorem 2.1, and is essentially the same as that of Alladi [2] or Pak [8].

Proof of Theorem 1.1. Replacing $q$ and $a$ by $q^{2}$ and -aq, respectively, (1.3) becomes

$$
\sum_{n=0}^{\infty} \frac{\left(-a q ; q^{2}\right)_{n}}{\left(q^{2} ; q^{2}\right)_{n}} z^{n}=\frac{\left(-a q z ; q^{2}\right)_{\infty}}{\left(z ; q^{2}\right)_{\infty}} .
$$

It is easy to see that the coefficient of $z^{n}$ on the left-hand side of (3.1) is equal to

$$
\sum_{\substack{\mu \in \mathcal{P}_{1} \\ \mu_{1} \leq 2 n}} q^{|\mu|} a^{\operatorname{odd}(\mu)},
$$

while the coefficient of $z^{n}$ on the right-hand side is equal to

$$
\sum_{\substack{\mu \in \mathcal{P}_{1} \\ \ell(\mu) \leq n}} q^{|\mu|} a^{\operatorname{odd}(\mu)} .
$$

The proof then follows from the involution $\sigma$ in the proof of Theorem 2.1.

Proof of Theorem 1.2. Replacing $q$ and $z$ by $q^{2}$ and $-z q$, respectively, (1.2) becomes

$$
\sum_{n=0}^{\infty} \frac{\left(-a z q ; q^{2}\right)_{n}}{\left(a ; q^{2}\right)_{n+1}} b^{n}=\sum_{n=0}^{\infty} \frac{\left(-b z q ; q^{2}\right)_{n}}{\left(b ; q^{2}\right)_{n+1}} a^{n} .
$$

It is easy to see that the coefficient of $a^{m} b^{n}$ on the left-hand side of (3.2) is equal to

$$
\sum_{\substack{\mu \in \mathcal{P}_{1} \\ \ell(\mu) \leq m \\ \mu_{1} \leq 2 n}} q^{|\mu|} z^{\operatorname{odd}(\mu)}
$$

while the coefficient of $a^{m} b^{n}$ on the right-hand side is equal to

$$
\sum_{\substack{\mu \in \mathcal{P}_{1} \\ \ell(\mu) \leq n \\ \mu_{1} \leq 2 m}} q^{|\mu|} z^{\operatorname{odd}(\mu)}
$$


By the involution $\sigma$ in the proof of Theorem 2.1, we have

$$
\sum_{\substack{\mu \in \mathcal{P}_{1} \\ \ell(\mu) \leq n \\ \mu_{1} \leq 2 m}} q^{|\mu|} z^{\operatorname{odd}(\mu)}=\sum_{\substack{\mu \in \mathcal{P}_{1} \\ \ell(\mu) \leq m \\ \mu_{1} \leq 2 n}} q^{|\mu|} z^{\operatorname{odd}(\mu)} .
$$

This completes the proof.

Replacing $q$ and $z$ by $q^{2}$ and $-z q$, respectively, (1.3) may be written as

$$
\begin{aligned}
& \sum_{k=0}^{n}(-1)^{k} \frac{\left(-q / z ; q^{2}\right)_{k}\left(-z q ; q^{2}\right)_{n-k}}{\left(q^{2} ; q^{2}\right)_{k}\left(q^{2} ; q^{2}\right)_{n-k}} q^{(2 m+1) k} z^{k} \\
& =\sum_{k=0}^{m}(-1)^{k} \frac{\left(-q / z ; q^{2}\right)_{k}\left(-z q ; q^{2}\right)_{m-k}}{\left(q^{2} ; q^{2}\right)_{k}\left(q^{2} ; q^{2}\right)_{m-k}} q^{(2 n+1) k} z^{k} .
\end{aligned}
$$

We will prove (3.4) combinatorially by first establishing the following two lemmas.

Lemma 3.1 For $m \geq 0$ and $n \geq 1$, we have

$$
\begin{aligned}
& \sum_{k=0}^{n}(-1)^{k} \frac{\left(-q / z ; q^{2}\right)_{k}\left(-z q ; q^{2}\right)_{n-k}}{\left(q^{2} ; q^{2}\right)_{k}\left(q^{2} ; q^{2}\right)_{n-k}} q^{(2 m+1) k} z^{k} \\
& =\sum_{\substack{(\lambda, \mu) \in \mathcal{P}_{2} \times \mathcal{P}_{1} \\
\ell(\lambda)+\ell(\mu) \leq n \\
\lambda_{\ell(\lambda)} \geq 2 m+1}}(-1)^{\ell(\lambda)} q^{|\lambda|+|\mu|} z^{\operatorname{odd}(\lambda)+\operatorname{odd}(\mu)} .
\end{aligned}
$$

Proof. It is easy to see that

$$
\begin{aligned}
\frac{\left(-q / z ; q^{2}\right)_{k}}{\left(q^{2} ; q^{2}\right)_{k}} z^{k} & =\sum_{\substack{\lambda \in \mathcal{D}_{\text {odd }} \\
\lambda_{1} \leq 2 k-1}} q^{|\lambda|} z^{k-\ell(\lambda)} \sum_{\substack{\mu \in \mathcal{P}_{\text {even }} \\
\mu_{1} \leq 2 k}} q^{|\mu|} \\
& =\sum_{\substack{\tau \in \mathcal{P}_{1} \\
\tau_{1} \leq 2 k}} q^{|\tau|} z^{k-\operatorname{odd}(\tau)},
\end{aligned}
$$

where $\tau=\lambda \cup \mu$.

By the involution $\sigma$ in the proof of Theorem 2.1, we have

$$
\sum_{\substack{\tau \in \mathcal{P}_{1} \\ \tau_{1} \leq 2 k}} q^{|\tau|} z^{k-\operatorname{odd}(\tau)}=\sum_{\substack{\tau \in \mathcal{P}_{1} \\ \ell(\tau) \leq k}} q^{|\tau|} z^{k-\operatorname{odd}(\tau)}
$$


Hence,

$$
\begin{aligned}
\frac{\left(-q / z ; q^{2}\right)_{k}}{\left(q^{2} ; q^{2}\right)_{k}} q^{(2 m+1) k} z^{k}=\sum_{\substack{\tau \in \mathcal{P}_{1} \\
\ell(\tau) \leq k}} q^{|\tau|+(2 m+1) k} z^{k-\operatorname{odd}(\tau)} \\
=\sum_{\substack{\lambda=\left(\lambda_{1}, \ldots, \lambda_{k}\right) \in \mathcal{P}_{2} \\
\lambda_{k} \geq 2 m+1}} q^{|\lambda|} z^{k-\operatorname{even}(\lambda)} \\
=\sum_{\substack{\lambda=\left(\lambda_{1}, \ldots, \lambda_{k}\right) \in \mathcal{P}_{2} \\
\lambda_{k} \geq 2 m+1}} q^{|\lambda|} z^{\operatorname{odd}(\lambda)}
\end{aligned}
$$

where $\lambda_{i}=\tau_{i}+2 m+1(1 \leq i \leq k)$.

Similarly, we have

$$
\frac{\left(-z q ; q^{2}\right)_{n-k}}{\left(q^{2} ; q^{2}\right)_{n-k}}=\sum_{\substack{\mu \in \mathcal{P}_{1} \\ \ell(\mu) \leq n-k}} q^{|\mu|} z^{\operatorname{odd}(\mu)}
$$

Therefore, the left-hand side of (3.5) is equal to

$$
\begin{aligned}
& \sum_{k=0}^{n}(-1)^{k} \sum_{\substack{\lambda=\left(\lambda_{1}, \ldots, \lambda_{k}\right) \in \mathcal{P}_{2} \\
\lambda_{k} \geq 2 m+1}} q^{|\lambda|} z^{\operatorname{odd}(\lambda)} \sum_{\substack{\mu \in \mathcal{P}_{1} \\
\ell(\mu) \leq n-k}} q^{|\mu|} z^{\operatorname{odd}(\mu)} \\
& =\sum_{\substack{(\lambda, \mu) \in \mathcal{P}_{2} \times \mathcal{P}_{1} \\
\ell(\lambda)+\ell(\mu) \leq n \\
\lambda_{\ell(\lambda) \geq 2 m+1}}}(-1)^{\ell(\lambda)} q^{|\lambda|+|\mu|} z^{\operatorname{odd}(\lambda)+\operatorname{odd}(\mu)},
\end{aligned}
$$

as desired.

Lemma 3.2 For $m \geq 0$ and $n \geq 1$, we have

$$
\sum_{\substack{(\lambda, \mu) \in \mathcal{P}_{2} \times \mathcal{P}_{1} \\ \ell(\lambda)+\ell(\mu) \leq n \\ \lambda_{\ell(\lambda)} \geq 2 m+1}}(-1)^{\ell(\lambda)} q^{|\lambda|+|\mu|} z^{\operatorname{odd}(\lambda)+\operatorname{odd}(\mu)}=\sum_{\substack{\mu \in \mathcal{P}_{1} \\ \ell(\mu) \leq n \\ \mu_{1} \leq 2 m}} q^{|\mu|} z^{\operatorname{odd}(\mu)} .
$$

Proof. Let

$$
\mathcal{B}:=\left\{(\lambda, \mu) \in \mathcal{P}_{2} \times \mathcal{P}_{1}: \ell(\lambda)+\ell(\mu) \leq n \text { and } \lambda_{\ell(\lambda)} \geq 2 m+1\right\}
$$

We will construct an involution $\phi$ on the subset

$$
\mathcal{B}_{m}:=\left\{(\lambda, \mu) \in \mathcal{B}: \lambda \neq 0 \text { or } \mu_{1} \geq 2 m+1\right\}
$$


of $\mathcal{B}$, with the properties that $\phi$ preserves $|\lambda|+|\mu|$ and $\operatorname{odd}(\lambda)+\operatorname{odd}(\mu)$ while sign-reversing $(-1)^{\ell(\lambda)}$.

For any $(\lambda, \mu) \in \mathcal{B}_{m}$, note that no even part of $\lambda$ is repeated while no odd part of $\mu$ is repeated. Define

$$
\phi((\lambda, \mu))= \begin{cases}\left(\left(\mu_{1}, \lambda_{1}, \lambda_{2}, \ldots\right),\left(\mu_{2}, \mu_{3}, \ldots\right)\right), & \text { if } \lambda_{1}<\mu_{1} \text { or } \lambda_{1}=\mu_{1}=2 s+1 \\ \left(\left(\lambda_{2}, \lambda_{3}, \ldots\right),\left(\lambda_{1}, \mu_{1}, \mu_{2}, \ldots\right)\right), & \text { if } \lambda_{1}>\mu_{1} \text { or } \lambda_{1}=\mu_{1}=2 s\end{cases}
$$

It is straightforward to verify that $\phi$ is an involution on $\mathcal{B}_{m}$ with the required properties. This proves that

$$
\sum_{(\lambda, \mu) \in \mathcal{B}_{m}}(-1)^{\ell(\lambda)} q^{|\lambda|+|\mu|} z^{\operatorname{odd}(\lambda)+\operatorname{odd}(\mu)}=0
$$

which implies (3.7).

Proof of Theorem 1.3. Combining Lemmas 3.1 and 3.2, we obtain

$$
\sum_{k=0}^{n}(-1)^{k} \frac{\left(-q / z ; q^{2}\right)_{k}\left(-z q ; q^{2}\right)_{n-k}}{\left(q^{2} ; q^{2}\right)_{k}\left(q^{2} ; q^{2}\right)_{n-k}} q^{(2 m+1) k} z^{k}=\sum_{\substack{\mu \in \mathcal{P}_{1} \\ \ell(\mu) \leq n \\ \mu_{1} \leq 2 m}} q^{|\mu|} z^{\text {odd }(\mu)} .
$$

By symmetry, we have

$$
\sum_{k=0}^{m}(-1)^{k} \frac{\left(-q / z ; q^{2}\right)_{k}\left(-z q ; q^{2}\right)_{m-k}}{\left(q^{2} ; q^{2}\right)_{k}\left(q^{2} ; q^{2}\right)_{m-k}} q^{(2 n+1) k} z^{k}=\sum_{\substack{\mu \in \mathcal{P}_{1} \\ \ell(\mu) \leq m \\ \mu_{1} \leq 2 n}} q^{|\mu|} z^{\text {odd }(\mu)} .
$$

The proof then follows from (3.3).

Acknowledgments. This work was done under the auspices of the National "973" Project on Mathematical Mechanization, and the National Science Foundation of China. The author would like to thank the referee for valuable comments.

\section{References}

[1] C. Adiga, B. C. Berndt, S. Bhargava, and G. N. Watson, Chapter 16 of Ramanujan's second notebook: theta-functions and q-series, Mem. Amer. Math. Soc., 53 (1985), $\mathrm{v}+85 \mathrm{pp}$.

[2] K. Alladi, A fundamental invariant in the theory of partitions, In: Topics in Number Theory (University Park, PA, 1997), Kluwer Academic Publishers, Dordrecht, 1999, pp. 101-113.

[3] G. E. Andrews, The Theory of Partitions, Cambridge University Press, Cambridge, 1998. 
[4] G. E. Andrews, J. Jiménez-Urroz, and K. Ono, $q$-series identities and values of certain L-functions, Duke Math. J., 108 (2001), 395-419.

[5] R. Chapman, Combinatorial proofs of $q$-series identities, J. Combin. Theory Ser. A, 99 (2002), 1-16.

[6] G. Gasper, Elementary derivations of summation and transformation formulas for q-series, In: Special Functions, q-Series and Related Topics (Toronto, ON, 1995), 55-70, Fields Inst. Commun., 14, Amer. Math. Soc., Providence, RI, 1997.

[7] G. Gasper and M. Rahman, Basic Hypergeometric Series, Encyclopedia of Mathematics and its Applications, Vol. 35, Cambridge University Press, Cambridge, 1990.

[8] I. Pak, Partition bijections, a survey, Ramanujan J., to appear.

[9] D. Stockhofe, Bijektive Abbildungen auf der Menge der Partitionen einer natürlichen Zahl, Bayreuth. Math. Schr. No. 10, (1982), 1-59. 